\title{
THE EFFECT OF IMPAIRED BALANCE AND FALL RISK ON QUALITY OF LIFE IN PATIENTS WITH DIABETIC PERIPHERAL NEUROPATHY
}

Paul Daniel V.K ${ }^{1}$, Syeda Umme Kulsum *2.

${ }^{1}$ Professor, RV College of Physiotherapy, affiliated to Rajiv Gandhi University of Health Sciences, Bangalore, Karnataka, India.

${ }^{* 2}$ RV College of Physiotherapy, affiliated to Rajiv Gandhi University of Health Sciences, Bangalore, Karnataka, India.

\section{ABSTRACT}

Background and Objectives: Diabetes mellitus (DM) has become the leading chronic disorders in nearly all countries due to urbanization, changing lifestyles and lack of physical activity. Neuropathy is the commonest and most debilitating complications of diabetes. Diabetic Peripheral Neuropathy (DPN) increases balance impairment, increasing fall risk. Consequences include decline in mobility, avoidance of activity, institutionalization and mortality; thus affecting one's Quality of Life (Quality of Life). Previous studies have focused on balance impairment and fall risk in DPN patients and also on QoL separately. Thus, the aim of this study is to find the effect of balance impairment and fall risk on QoL.

Methods: A correlational study was done on 30 DPN patients, selected by purposive sampling technique. Subjects were screened for neuropathy using Toronto Clinical Neuropathy Scoring System (TCSS) and those fulfilling the inclusion criteria, underwent balance and fall risk assessment by Berg Balance Scale (BBS) and Timed Up and Go (TUG) Test respectively. Following which, neuropathy specific quality of life questionnaire (NeuroQoL) was administered to evaluate their QoL. Results obtained were statistically analysed using SPSS 16.0, MS Word and MS Excel. Pearson's correlation was used to find the correlation between variables.

Results: The results showed statistically significant correlation between all the variables. Correlation between BBS and reduction in QoL ( $r=-0.540, p=0.002)$, TUG and reduction in QoL $(r=0.531, p=0.003)$.

Conclusion: In conclusion, physical limitations such as balance impairment and greater fall risk significantly reduces DPN patient's QoL.

KEY WORDS: Diabetic Peripheral Neuropathy, Balance Impairment, Fall Risk, Quality of Life.

Address for correspondence: Syeda Umme Kulsum (MPT pursuing), Student, RV College of Physiotherapy, affiliated to Rajiv Gandhi University of Health Sciences, Bangalore, Karnataka, India.

E-Mail: syedaummekulsumrazvi@gmail.com

Access this Article online

Quick Response code

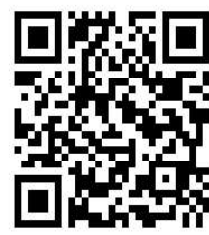

DOI: $10.16965 /$ ijpr.2019.172

Journal Information

International Journal of Physiotherapy and Research

ICV for 2016 ISSN (E) 2321-1822 | ISSN (P) 2321-8975

https://www.ijmhr.org/ijpr.html

DOI-Prefix: https://dx.doi.org/10.16965/ijpr

\section{Article Information}

Received: $10 \mathrm{Jul} 2019$

Peer Review: 10 Jul 2019

Revised: None
Accepted: 14 Aug 2019

Published (O): 11 Sep 2019

Published (P): 11 Oct 2019

\section{INTRODUCTION}

Diabetes mellitus (DM) is a metabolic disorder which is characterized by the presence of chronic hyperglycemia, resulting either from immune mediation (type 1), insulin resistance (type 2 ), gestation (gestational) or other causes (genetic, environmental defects, drugs or any infections)
[1]. The chronic hyperglycemia is associated with long term damage, dysfunction and organ failure mainly of the eyes, kidneys, nerves, heart and blood vessels [2]. DM is one of the largest global public health emergencies of the twentyfirst century [3]. It is one of the most common chronic diseases in nearly all countries, and 
continues to increase in numbers and significance, as economic development and urbanization is leading to changing lifestyles, characterized by reduced physical activity and increased obesity [4]. The number of people with diabetes has risen from 108 million in 1980 to 422 million in 2014 the global prevalence of diabetes among adults over eighteen years of age has risen from $4.7 \%$ in 1980 to $8.5 \%$ in 2014 [5]. In India, there were over seventy two million cases of diabetes with the prevalence rate of $8.8 \%$ in 2017[6]. Type 2 diabetes mellitus (T2DM), once known as adult onset or non-insulin dependent diabetes, is a chronic condition that affects the way the body metabolizes sugar, body's most important source of fuel. With T2DM, body either resists the effects of insulin (a hormone that regulates the movement of sugar into the cells) or doesn't produce enough insulin to maintain a normal glucose level [7]. It makes up about $85-90 \%$ of all cases [8].

Neuropathies are one of the most common complications of DM, with a prevalence of approximately $13.46 \%$ [6]. Diabetic peripheral neuropathy (DPN) is characterized by the presence of signs and symptoms of peripheral nerve dysfunction in diabetic patients, after excluding the non-diabetic cause [9].

The pathogenesis of DPN is mainly due to small vessel disease (microangiopathy). Diabetic peripheral neuronal damage is of two types- mostly due to axonal damage and a lesser extent due to demyelination [2]. DPN affects the proximal and distal peripheral sensory and motor nerves and also the autonomic nervous system [10]. Small and large fibre neuropathies occur early in DPN. The small fibre neuropathies primarily manifests as pain, burning sensation and paraesthesia in lower limbs during the early phase of diabetes. Large fibre neuropathy in diabetes occurs with signs of neuropathy, manifesting as impaired vibration or joint sense. The numbness and weakness in lower limbs occur much later in the illness. These symptoms usually begins at the tip of toes or fingers and gradually spread upwards distributed in "Stocking and Glove manner"[11]. These symptoms are generally worst at night and disturb sleep $[12,13]$.

A number of risk factors are associated with the onset of DPN and the most important ones include poor glycemic control and duration of diabetes [2]. Toronto Clinical Scoring System (TCSS) is a sensitive scoring system to diagnose the presence and severity of diabetic peripheral neuropathy and it correlates well with underlying structural damage in peripheral nerves as shown by the loss of myelinated nerve fibers. The severity of neuropathy is based on 3 domain scores that is; symptom scores, reflex scores and sensory test scores; the score range being $0-19$. It is used as an inexpensive bedside screening tool in clinics and in clinical research trials $[11,25]$.

DPN affects nearly $60-70 \%$ of the individuals with diabetes and leads to increased risk of fall $[14,15]$. DPN may compromise balance during daily activities [16]. Berg Balance Scale (BBS) is a gold standard used to objectively determine a patient's ability (or inability) to safely balance during a series of predetermined tasks of Activities of Daily Living (ADL). It is a 14-item list with each item consisting of five-point ordinal scale ranging from $0-4$. However, it does not include the assessment of gait [17]. A fall is preceded by loss of balance, which may be recoverable in some individuals, but requires rapid responses and good strength of the lower limb muscles [18]. DPN gradually affects distal muscle strength and deteriorates normal walking function. Alterations in peripheral nerves is the chief contributor of balance impairment, gait instabilities and falls [9]. However, the risk of falls increases considerably with age and comorbidities and in DPN patients than in diabetics without DPN [19]. Nevertheless, the more likely an individual is to loose balance, the more likely they will at some point experience a fall [16]. The Timed Up and Go test (TUG) is a simple test used to assess a person's functional mobility and requires both static and dynamic balance. It quantifies the risk of fall of an individual. TUG was developed from a more comprehensive test called the Get Up and Go test [20].

Patients with DPN have a five-fold increased risk of falling and the consequences include decline in mobility, avoidance of activity, institutionalization and mortality [21]. The economic consequences of diabetic foot problems are major, both to society as well as to the patients and 
their families [2]. DPN has a profound effect on patient's quality of life (QoL) affecting various physical and psychosocial aspects of their lives [22]. NeuroQoL (Quality of Life in Neurological Disorders) is a measurement system that evaluates and monitors the physical, mental and social effects experienced by adults and children living with neurological conditions. It is a multidimensional scale developed by Vileikyte et al (2003) to assess QoL of diabetic patients with peripheral neuropathy. It is a questionnaire comprising questions related to neuropathy symptoms as well as psychosocial functioning in six domains. The impact of each item is measured by marking against the scale [23].

Problem Definition: Since DM has become the leading chronic disorders in nearly all countries due to urbanization, changing lifestyles and lack of physical activity. Neuropathy is one of the commonest and most debilitating complications of diabetes, the risk of which increases with time and poor glycemic control. Not only the patient, but it also traumatizes their families and societies in the form of foot ulcers and amputations, and increased healthcare expenditure. Neuropathy increases balance impairment and thereby increasing the risk of falls. It leads to increased institutionalization, reduced mobility and participation leaving a negative impact on one's quality of life. But, there is a lack of evidence to know the effect of balance impairment and fall risk on one's quality of life in patients with DPN. Therefore, quantifying balance impairment while performing activities of daily living (ADLs) may be considered as one of the closest proxies for determining the risk of falls and thus estimating its impact on the quality of life in patients with diabetic peripheral neuropathy. Thus correlating impaired balance and fall risk on quality of life in patients with DPN.

\section{METHODS}

Study Design: Correlational study.

Study Setting: Jnana Sanjeevini Hospital, Bangalore and Out Patient Department of R.V College of Physiotherapy.

Study Duration: six months.

Sampling Technique: Purposive Sampling Technique.
Sample Size Estimation: Sample size of $n=30$ was determined through power calculation based on prevalence rate of $p=13.46 \%$ for T2DM patients with DPN, obtained from previous studies. Eliminating the type 1 error á $=5 \%$, type 2 error â $=20 \%$ and mean standard error $d=20 \%$, substituting in the formula:

$$
n=\left(z \frac{\alpha}{2}+Z \beta\right)^{2} p q \div \mathrm{d}^{2} .
$$

Inclusion Criteria: (1) Subjects willing to participate and sign the written informed consent. (2)Age group between $40-60$ years. (3) History of T2DM for e" 5 years. (4) Toronto CSS score ranging between 6-11 points. (5) Ability to walk independently.

Exclusion Criteria: (1) Inability to provide accurate medical history. (2) History of visual (including diabetic retinopathy) or vestibular impairment. (3) History of neuropathies of the non-diabetic cause. (4) Subjects under any neuro-protective drugs. (5) Presence of any other neurological condition which compromise balance. (6) Musculoskeletal ailments of lower extremity leading to pain and instability. (7) Presence of foot ulcers and / or any amputation.

Materials and Equipments: TCSS sheet, BBS scoring sheet, TUG test scoring sheet, NeuroQoL questionnaire form, two chairs (one with arm rest and one without arm rest), stopwatch or a timer, stepper, measuring tape, reflex hammer, tuning fork (128hz), 10g monofilament, hot and cold test tubes, clear walkway of $3 \mathrm{~m}, 15 \mathrm{ft}$, stationeries.

Ethical clearance: Permission to carry out the study was obtained from the concerned authorities of the above mentioned institutions. Ethical clearance from the Ethical Committee Review Board was obtained before carrying out the study. The test procedure was explained and signed informed written consent was obtained from each subject on their approval.

Procedure: Subjects were recruited as per the inclusion criteria. Test procedure was explained and signed informed written consent was obtained from each subject on their approval. Materials and equipments required for the procedure were arranged prior to the test. Demographic data of the subjects was collected 
and recorded. Data regarding the type of diabetes, history of diabetes, history of peripheral neuropathy, treatment history and any known co-morbidities and their undergoing treatment procedures was obtained from the patient's medical records. Prior to the test, subjects were screened for the severity of DPN based on the scores of TCSS.

Balance and Fall Risk Assessment: Berg Balance Scale-Subjects were given clear instructions to perform the various tasks illustrated in the BBS one after another, under supervision. The 14 tasks included- sit to stand, sitting unsupported, standing unsupported, stand to sit, transfers, standing with eyes closed, standing with narrow base of support, forward reaching, retrieving objects from floor, turning to 180 degrees and 360 degrees, stool stepping and tandem standing. The whole procedure took 15 to $20 \mathrm{~min}$ to administer. Each task was rated from 0 (unable) to 4 (independent) based on their performance. Test results were interpreted as follows: $41-56$ = low fall risk, $21-40=$ medium fall risk, $0-20=$ high fall risk $[21,26]$.

Timed Up and Go test- Quantification of balance and functional mobility was achieved by TUG test. $3 \mathrm{~m}$ distance was marked from the chair, on an even terrain in a well-lit environment. Subjects were made to sit in the chair with armrest and their backs supported against the backrest. Clear verbal instructions were given to - stand up from the chair, walk the distance as quickly and as safely as possible, cross the line marked on floor, turn around, walk back as quickly and as safely as possible, and sit down. The timer was started once the examiner said ' $G O$ ' and it was stopped once the subject sat down comfortably in the chair with his/her arms and back supported. One practice trial and two test trials were conducted. During the test, examiner was standing close to the subjects, to steady them if necessary. Test results were interpreted as follows: $\leq 4-8 \mathrm{sec}=$ normal, $9-10=$ low risk of fall, $11-13=$ medium risk of fall, $\geq 13.5 \mathrm{sec}=$ high risk of fall.[21, 28]

Quality Of Life Assessment: NeuroQoL- A NeuroQoL questionnaire was given to the subjects and instructed to fill it according to their perception of symptoms. The examiner translated the questions of the questionnaire in the language best understood to the patient, for those who did not understand English. The questionnaire comprised questions related to neuropathy symptoms as well as psychosocial functioning in six domains such as - pain and paraesthesia, reduced or loss of sensation, diffused sensory-motor symptoms, activity limitation, interpersonal problems and emotional burden. It has a five point scale ranging from 1 (never) to 5 (always). The impact of each of these items was measured by asking the subjects to mark against each of these items on another scale ranging from 1 (not at all) to 3 (a lot). Weighted score for each item on the respective domain was calculated by multiplication of the value against each item on 1-5 scale with the importance of it on 1-3 scale. Total value of scores in each domain was calculated by the mean of weighted items on the respective domain, with highest value corresponding to poor health related quality of life (HRQoL) [23].

Statistical Analysis: The statistical software SPSS 16.0 was used for the analysis of the data and MS- Word 2013 and MS-Excel 2013 have been used to generate graphs, tables, etc. Descriptive and inferential statistical analysis has been carried out. Results on continuous measurements are presented on Mean \pm SD (Min-Max) and on categorical measurements are presented in Number (\%). Pearson's correlation coefficient was applied with a confidence interval set at $95 \%$ between BBS and Neuro QoL and between TUG and Neuro QoL. Significance was assessed at $5 \%$ level of significance.

Correlation range:

- Weak correlation (anything Â \pm 0.25 )

- Intermediate correlation (between \pm 0.26 to \pm 0.75 )

- Strong correlation (between \pm 0.76 to \pm 0.99 )

- Perfect correlation $( \pm 1)$.

\section{RESULTS}

Total sample size of 30 (100\%) subjects, out of which there were $10(33 \%)$ male subjects and 20 (67\%) female subjects in this study (table 1). The Mean \pm SD of age distribution was found to be $52.26 \pm 4.94$ (table 2). $43.33 \%$ of the subjects had Mild DPN and $56.67 \%$ of them had Moderate DPN (Table 3 and fig 1). 
Table 1: Gender Distribution.

\begin{tabular}{|c|c|c|c|c|}
\hline Age & Male & Female & Total & Percentage \\
\hline $40-45$ & 3 & 0 & 3 & $10 \%$ \\
\hline $46-50$ & 1 & 8 & 9 & $30 \%$ \\
\hline $51-55$ & 3 & 7 & 10 & $33.33 \%$ \\
\hline $56-60$ & 3 & 5 & 8 & $26.67 \%$ \\
\hline
\end{tabular}

Table 2: Age Distribution.

\begin{tabular}{|c|c|c|}
\hline Gender & Frequency & Percentage \\
\hline Male & 10 & $33 \%$ \\
\hline Female & 20 & $67 \%$ \\
\hline Total & 30 & $100 \%$ \\
\hline
\end{tabular}

Table 3: Neuropathy Distribution

\begin{tabular}{|c|c|c|}
\hline TCSS (Neuropathy) & Frequency & $\%$ \\
\hline $6-8$ (Mild) & 13 & $43.33 \%$ \\
\hline 9-11 (Moderate) & 17 & $56.67 \%$ \\
\hline
\end{tabular}

Fig. 1: Neuropathy Distribution.

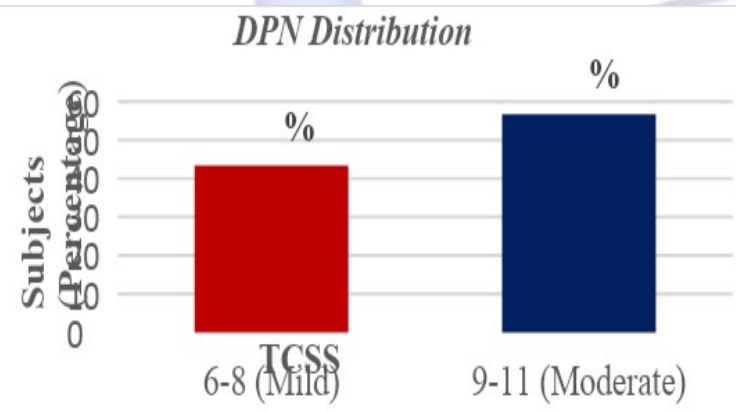

Fig. 2: Severity of DPN and Balance impairment

Neuropathy and Balance

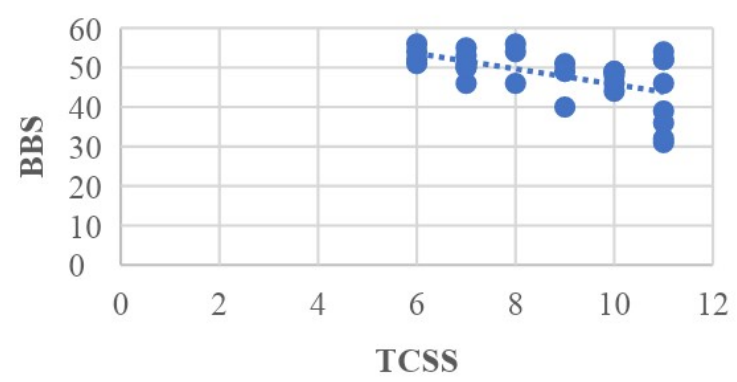

Fig. 3: Severity of DPN and fall risk Neuropathy and Fall risk

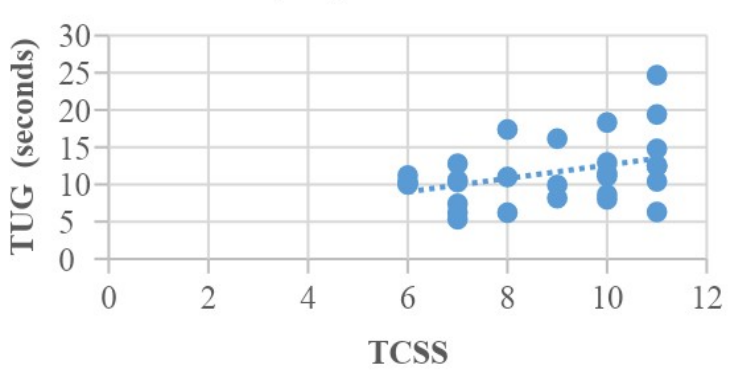

Fig. 4: Balance and Reduction in QoL

\section{Balance and Reduction in QoL}

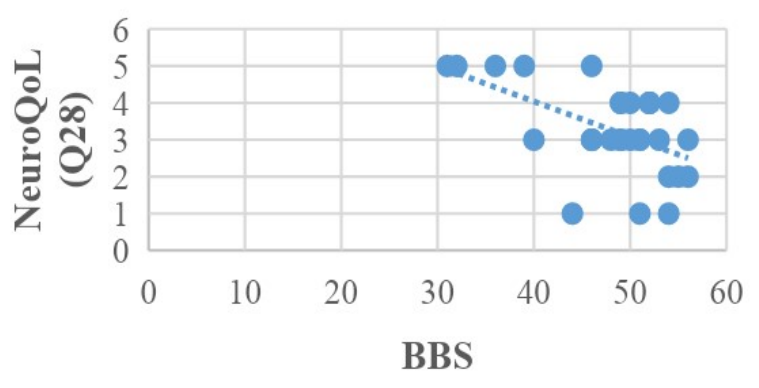

Fig. 5: Fall risk and Reduction in QoL.

\section{Fall risk and Reduction in}

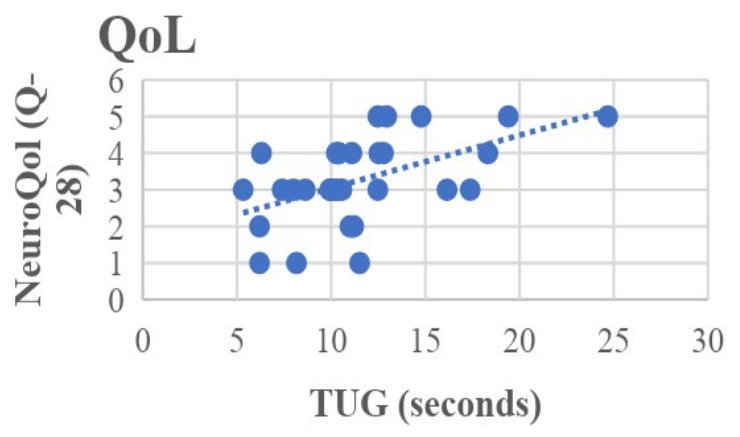

Table 4: Correlation of the Data.

\begin{tabular}{|c|c|c|c|c|c|c|c|c|c|}
\hline (a) BBS & BBS & QA & QB & QC & QD & QE & QF & Q28 & Q29 \\
\hline Pearson correlation (r) & 1 & -0.619 & -0.603 & -0.762 & -0.428 & -0.645 & -0.663 & -0.54 & 0.588 \\
\hline p-value & & 0 & 0 & 0 & 0.018 & 0 & 0 & 0.002 & 0.001 \\
\hline (b) TUG & TUG & QA & QB & QC & QD & QE & QF & Q28 & Q29 \\
\hline Pearson correlation (r) & 1 & 0.56 & 0.463 & 0.567 & 0.587 & 0.623 & 0.475 & 0.531 & -0.548 \\
\hline p-value & & 0.001 & 0.01 & 0.001 & 0.001 & 0 & 0.008 & 0.003 & 0.002 \\
\hline
\end{tabular}

**Correlation is significant at the 0.01 level (2-tailed) The above table 4 a shows the correlation between balance impairment and neuropathy specific quality of life of individuals with DPN. Table $4 \mathrm{~b}$ shows the correlation between fall risk and neuropathy specific quality of life of DPN patients. QA TO QF shown in the above table corresponds to different domains of the self-
*Correlation is significant at the 0.05 level (2-tailed). administered questionnaire NeuroQoL, which are as follows- QA (pain and paraesthesia), QB (loss of sensations) QC (diffused sensorymotor symptoms), QD (activity limitation), QE (interpersonal problems) and QF (emotional burden). While Q28 and Q29 corresponds to Reduction in QoL and Overall QoL, respectively. 
Table 4a shows a significant negative correlation between balance impairment and QoL. The correlation was strongly negative for $Q C$ (diffused sensory-motor symptoms). However, the correlation between BBS and Q28 ( $r=-0.540$, $\mathrm{p}=0.002$ ) was significantly negative, which suggests that higher the balance maintaining capacity, lesser is the reduction in QoL in DPN patients. Conversely, the correlation between BBS and Q29 ( $r=0.588, p=0.001$ ) was significantly positive, suggestive of higher the balance maintaining capacity, better is the overall QoL of DPN patients.

Table $4 \mathrm{~b}$ shows a significant positive correlation between fall risk and QoL. The correlation between TUG and Q28 ( $r=0.531, p=0.003$ ) was significantly positive, which suggests that higher the fall risk, greater is the reduction in QoL in DPN patients. Conversely, the correlation between TUG and Q29 ( $r=-0.548, p=0.002$ ) was significantly negative, suggestive of higher the fall risk, poorer is the overall QoL of DPN patients.

\section{DISCUSSION}

Peripheral neuropathy is the most common complications of diabetes which has an extensive impact on patient's health and health related quality of life. Falls are marked as a dangerous health issue in DPN especially in the geriatric population [9].

The findings of this study are consistent with previous studies which observed that the balance impairments and fall risk increase with the increasing severity of DPN [3]. Previous studies have proved that balance impairments in DPN patients occurs due to deficits of systems that control balance. Interruption of the afferent and efferent neuron function leads to reduced proprioception and tactile sensation with deterioration of somatosensory, visual and vestibular systems which in-turn causes postural instability and larger postural sway. Muscle strength reduction in postural and lower limb muscles, associated with relatively high glucose levels may contribute to impaired balance [9].

A study conducted by Menz et al (2004) suggests, the diminished sensory feedback with further contributions of vision impairments, muscle weakness, and lack of neuromuscular control of distal joints in neuropathic patients results in increased risk of falls.

In the present study it was found that both balance impairment and fall risk had a statistically significant strong correlation with the reduction in patient's neuropathy specific QoL, which is consistent with the study findings of Porjan et al. (2012) which stated that patients with DM have statistically significant impairment of all aspects of QoL. In the present study, it was also found that there exists a statistically significant positive correlation between balance ability and QoL and a statistically significant negative correlation between fall risk and QoL. The findings of present study observed that the correlation between balance ability and the diffused sensory-motor symptoms aspect of QoL was the strongest, which is in agreement with the study conducted by Dr. AS Ahmed (2017).

The study conducted by Irshad et al (2017), regarding the diffused sensory-motor symptoms, found that $30 \%$ of people with DPN, experience muscle weakness, loss of ankle reflexes, and decreased balance, coordination and gait control and indicated that weakness in the feet was considered the highest problem, followed by instability when standing or walking. Difficulty in maintaining the balance may be related to the instability in the muscles [22]. The balance and gait characteristics change as one's age progress and the presence of DPN in elderly population it plays a significant role in incidence of falls [35]. Static as well as dynamic balance are both affected in DPN patients [22]. Vileikyte et al. (2009) showed that impaired balance had the strongest association with the depressive symptoms which were due to perceptions of diminished value of the self, as a result of inability to perform family roles [36].

Al-Shehri et al (2014) reported that the presence of DPN significantly affects patient's QoL, especially physical function. The findings of present study are supported by the previous studies which states that the impact of physical limitations from DPN (due to impaired balance and high fall risk) has the same prevalent effect on psychosocial well-being (health related QoL) [22]. 


\section{CONCLUSION}

Balance impairment and fall risk significantly increases with the increasing severity of neuropathy. This study concluded that, physical limitations such as balance impairment and greater fall risk has significantly reduced patient's QoL. It leaves a negative impact on their QoL, by reducing their participation in ADLs, leisure activities and limiting their societal participation. All of these provoke depressive symptoms in DPN further reducing their activity level and thus reducing their QoL.

Future Scope: As we can see that balance impairment and fall risk significantly reduces patient's QoL, interventional strategies must be developed to improve balance and prevent falls among DPN patients to improve their QoL.

Limitations: Glycemic index of the patients was not considered. NeuroQoL questionnaire is more subjective evaluation of QoL. An objective evaluation of QoL would give us a better understanding of their neuropathy related QoL.

\section{ABBREVIATIONS}

DM - Diabetes Mellitus

T2DM - Type 2 Diabetes Mellitus

DPN - Diabetic Peripheral Neuropathy

TCSS or Toronto CSS - Toronto Clinical Scoring System

BBS - Berg Balance Scale

ADL or ADLs - Activities of Daily Living

TUG - Timed Up and Go

QoL - Quality of Life

NeuroQoL - Neuropathy specific Quality of Life

HRQoL - Health Related Quality of Life

SD - Standard Deviation

\section{ACKNOWLEDGEMENTS}

We would like to acknowledge the institutions (Jnana Sanjeevini Hospital, Bangalore and RV College of Physiotherapy, Bangalore) for their kind cooperation for giving us the opportunity to conduct our study in their esteemed institutions and also we would like to acknowledge all the participants of the study for their kind cooperation and participation in the study.

\section{Conflicts of interest: None}

\section{REFERENCES}

[1]. Habtamu Wondifraw Baynest. Classification, Pathophysiology, Diagnosis and Management of Diabetes Mellitus. J Diabetes Metab. 2015; 6(5).
[2]. Thomas Paul, Nihal Thomas. Peripheral neuropathy. In: Nihal Thomas, Kanakamani Jeyaraman, HS Asha, Jachin Velavan, K Senthilvasan. A Practical Guide to DIABETES MELLITUS, 2012, 6/e. Jaypee Brothers Medical Publishers, India.

[3]. Bogdan Timar, Romulus Timar, Laura Gaita, Cristian Oancea, Codrina Levai, Diana Lungeanu. The Impact of Diabetic Neuropathy on Balance and on the Risk of Fall in Patients with Type 2 Diabetes Mellitus: A cross-sectional study. PLoS ONE. 2016; 11(4).

[4]. David R Whiting, Leonor Guariguata, Clara Weil, Jonathan Shaw. Global Estimates of the Prevalence of Diabetes for 2011 and 2030. IDF Diabetes Atlas. 2011. Elsevier

[5]. World Health Organisation Diabetes fact sheet (2017). http://www.who.int/mediacentre/ factsheets/fs312/en/. ( seen on 16.03.2018)

[6]. India - International Diabetes Federation. https:// www.idf.org $>$ members $>94$-india. (seen on 16.03.2018)

[7]. Type 2 diabetes- symptoms and causes Mayo clinic. (seen on 16.03.2018)

[8]. NCD Alliance- Diabetes. 2014 hungary. https:// ncdalliance.org/diabetes. (seen on 16.03.2018)

[9]. Amirah Mustapa, Maria Justine, Nadia Mohd Mustafa, Nursuriati Jamil, Haidzir Manaf. Postural control and gait performance in the diabetic peripheral neuropathy: a systematic review. BioMed Research International. 2016.

[10]. A.I.Vinik. Advances in diabetes for the millennium: new treatments for diabetic neuropathies. Medscape General Medicine. 2004; 6(3): 13.

[11]. D Udayashankar, Sarah S Premraj, K Mayilananthi, Vishwanath Naragond. Applicability of Toronto Clinical Neuropathy Scoring and its Correlation with Diabetic Peripheral Neuropathy- A Prospective Cross Sectional Study. Journal of Clinical and Diagnostic Research. 2017; 11(2).

[12]. Robert J Tanenberg. Diabetic Neuropathy: Painful or Painless. Hospital Physician. 2009 (seen on 18.03.2018)

[13]. Jong Chul Won,Yong-Jin Im, Ji-Hyun Lee, Chong Hwa Kim, Hyuk Sang Kwon, Bong-Yun Cha et al. Clinical Phenotype of Diabetic Peripheral Neuropathy and Relation to Symptom Patterns: Cluster and Factor Analysis in Patients with Type 2 Diabetes in Korea. Journal of Diabetes Research. 2017.

[14]. Stephen D. Jernigan, Patricia S. Pohl, Jonathan D. Mahnken, Patricia M. Kluding. Diagnostic Accuracy of Fall Risk Assessment Tools in People with Diabetic Peripheral Neuropathy. American Physical Therapy Association. 2012; 92: 1461-1470.

[15]. Carmella Wint, Joann Jovinelly. Everything you should know about diabetic neuropathy. Health line. 2017. https://www.healthline.com/health/ type-2-diabetes/diabetic-neuropathy. (seen on 18.03.2018)

[16]. Brown SJ, Handsaker JC, Bowling FL, Boulton AJ, Reeves ND. Diabetic Peripheral Neuropathy Compromise Balance during Daily Activities. Diabetes care. $2015 ; 38(6): 1116-22$. 
[17]. http://www.strokecenter.org \trials \scales \berg.pdf (seen on 16.11.2018).

[18]. Pijnappels M, Reeves ND, Maganaris CN, van Dieen $\mathrm{JH}$. Tripping Without Falling; Lower Limb Strength, a Limitation for Balance Recovery and Target for Training in the Elderly. J Electromyography kinesiology. 2008; 18(2): 188-196.

[19]. Mark W. Powell, Dale H. Carnegie, Thomas J. Burke. Reversal of diabetic peripheral neuropathy with phototherapy (MIRE'M ) decreases falls and the fear of falling and improves activities of daily living in seniors. Age and Ageing. 2006; 35: 11-16.

[20]. http://www.Timed-Up-and-Go-Test scoring sheet (seen on 16.11.2018)

[21]. Hewston P, Deshpande N. Falls and Balance Impairments in Older Adults with Type 2 Diabetes: Thinking Beyond Diabetic Peripheral Neuropathy. Can J Diabetes. 2016; 40(1):6-9.

[22]. Dr.Amal Samir Ahmed. Assessment of Quality of Life of Patients with Diabetic Peripheral Neuropathy. IOSR Journal of Nursing and Health Science. 2017; 6(5): 37-46.

[23]. Loretta Vileikyte et al. The Development and Validation of a Neuropathy-and Foot Ulcer-Specific Quality of Life Instrument. Diabetes Care. 2003; 26(9): 2549-2555.

[24]. Mamta Jaiswal et al. Prevalence of and Risk Factors for Diabetic Peripheral Neuropathy in Youth with Type 1 and Type 2 Diabetes: SEARCH for Diabetes in Youth Study. Diabetes Care. 2017; 40: 1226-1232.

[25]. Vera Bril, Bruce A Perkins. Validation of the Toronto Clinical Scoring System for Diabetic Polyneuropathy. Diabetes Care. 2002; 25(11): 2048-2052.

[26]. Neuls Patrick D et al. Usefulness of Berg Balance Scale to Predict Falls in the Elderly. Journal of Geriatric Physiotherapy. 2011; 34(1): 3-10.

[27]. Mark A Davis, Sinead Brophy, Rhys Williams, Ann Taylor. The Prevalence, Severity, and Impact of Painful Diabetic Peripheral Neuropathy in Type 2 Diabetes Mellitus. Diabetes care. 2006; 29(7): 1518-1522.
[28]. Shumway-Cook A, Brauer S, Woollacott M. Predicting the Probability for Falls In Community-Dwelling Older Adults Using the Timed Up \& Go Test. Phys Ther. 2000; 80: 896-903.

[29]. Aaron Charles Lobo, Peter George, Kavina Marian Fernandes. An assessment of the Patterns and severity of diabetic neuropathy using the modified - Toronto Clinical Neuropathy Score in recently detected diabetics. International Journal of Biomedical Research. 2017; 8(05): 266-270.

[30]. Lori.J.Tuttle et al. Early Onset Physical Frailty in Adults with Diabesity and Peripheral Neuropathy. Canadian Journal of Diabetes. 2018; 42(5):478-483.

[31]. Muideen.T et al. Effect of Diabetes on Peripheral Neuropathy in an Adult US Population. American Diabetes Association. 2018; 67(1).

[32]. Amy Muchna et al. Association with Incident Falls, Frailty, Syndrome and Sensor-derived Gait, Balance and Physical Activity Measures. Journal of the American Podiatric Medical Association. 2018; 108(2).

[33]. Ilania Zivi et al. Effectiveness of Aquatic versus Land Physiotherapy in the Treatment of Peripheral Neuropathies: A Randomized Controlled Trial. 2017.

[34]. HB Menz Et Al. Walking Stability And Sensory Motor Function In Older People With Diabetic Peripheral Neuropathy. Archieves Of Physical Medicine And Rehabilitation. 2004; 85(2).

[35]. Irshad Et Al. Balance Training In Diabetic Peripheral Neuropathy: A Narrative Review. JSM Diabetology And Management. 2017; 2(1): 1002.

[36]. Vileikyte, L. Et Al. Predictors Of Depressive Symptoms In Persons With Diabetic Peripheral Neuropathy: A Longitudinal Study. Diabetologia. 2009; 52(7):1265-1273.

\footnotetext{
How to cite this article:

Paul Daniel V.K, Syeda Umme Kulsum. THE EFFECT OF IMPAIRED BALANCE AND FALL RISK ON QUALITY OF LIFE IN PATIENTS WITH DIABETIC PERIPHERAL NEUROPATHY. Int J Physiother Res 2019;7(5):3239-3246. DOI: 10.16965/ijpr.2019.172
} 